\title{
STABLE RANK OF SUBALGEBRAS OF THE BALL ALGEBRAS
}

\author{
RUDOLF RUPP \\ (Communicated by Paul S. Muhly)
}

\begin{abstract}
We show that every subalgebra (in the algebraic sense) of $A^{1}\left(B_{n}\right)$ satisfying the weak Nullstellensatz has stable rank less than or equal to $n$. The results are sharp for $n \leq 2$.
\end{abstract}

\section{INTRODUCTION}

The concept of the stable rank of a ring, introduced by H. Bass [1], has been very useful in treating some problems in algebraic $K$-theory. $G$. Corach and F. D. Suárez succeeded in calculating the stable rank of finitely generated Banach algebras, see $[2,3,4]$. Among them are the ball algebras $A\left(B_{n}\right)$. The proof in [4] makes use of a deep algebraic-topological argument-the so-called ArensTaylor-Novodvorski theory. It is the aim of this paper to present fairly simple proofs of inequalities for the stable rank of subalgebras of $A\left(B_{n}\right)$ (see Theorem 3.1 and Theorem 3.2).

Especially for the cases when $n \leq 2$, we do not only obtain inequalities, but equality. Thus for $n=1$ some results in $[7,8]$ are reproved.

The proofs make use only of elementary knowledge of functions of several complex variables.

1.

It is well known that the group of units in Banach algebras is open. Unfortunately, this feature is lost in the general case of a topological algebra. This leads to the following definitions:

A topological algebra $A$ is called a $Q$-algebra if the set of units $A^{X}$ is open in $A$.

In this paper we consider complex, commutative $Q$-algebras with unit element denoted by 1 .

Given a $Q$-algebra $A$, an element $a \in A^{n}$ is called unimodular if there exists

Received by the editors October 2, 1989.

1980 Mathematics Subject Classification (1985 Revision). Primary 46J15; Secondary 18F25. 
$b \in A^{n}$ such that

$$
\langle b, a\rangle:=\sum_{i=1}^{n} b_{i} a_{i}=1 .
$$

We denote by $U_{n}(A)$ the set of unimodular elements of $A^{n}$. We say that $a=\left(a_{1}, \ldots, a_{n}\right) \in U_{n}(A)$ is reducible, if there exist $x_{1}, \ldots, x_{n-1}$ in $A$ such that

$$
\left(a_{1}+x_{1} a_{n}, \ldots, a_{n-1}+x_{n-1} a_{n}\right) \in U_{n-1}(A) .
$$

The stable rank of $A$, denoted by $\operatorname{sr}(A)$, is the least integer $n$ such that every $a \in U_{n+1}(A)$ is reducible.

We need the following two results on the stable rank of $Q$-algebras [2, Theorem 3.8; 2, Lemma 3.7]. (The proofs given there are valid for $Q$-algebras. Of course sequences have to be replaced by nets.)

Proposition 1.1. Let $A$ be a Q-algebra and let $\gamma:[0,1] \rightarrow A^{n}$ be a path such that every vector $(\gamma(t), a)$ is unimodular and $(\gamma(0), a)$ is reducible. Then $(\gamma(1), a)$ is reducible, too.

Proposition 1.2. Let $\left(a_{k}, b\right)$ be a sequence of unimodular $(n+1)$-vectors in the $Q$-algebra $A$ and suppose that $a_{k}$ tends to $a$. Then $(a, b)$ is reducible if it is unimodular.

2.

Let $n$ be a fixed positive integer. The open unit ball will be denoted by $B_{n}$. Thus $B_{n}$ consists of all $z \in \mathbf{C}^{n}$ with $|z|^{2}:=\sum_{j=1}^{n}\left|z_{j}\right|^{2}<1$.

The boundary of $B_{n}$ is the sphere $S_{2 n-1}$, i.e.

$$
S_{2 n-1}=\left\{z \in \mathbf{C}^{n}: \sum_{j=1}^{n}\left|z_{j}\right|^{2}=1\right\} \text {. }
$$

The ball algebra $A\left(B_{n}\right)$ is the class of all $f: \bar{B}_{n} \rightarrow \mathbf{C}$ that are continuous on the closed ball $\bar{B}$ and holomorphic in its interior $B$. Moreover, we are interested in the subalgebras $A^{m}\left(B_{n}\right):=A\left(B_{n}\right) \cap C^{m}\left(\bar{B}_{n}\right)$ and $A^{\infty}\left(B_{n}\right):=$ $\bigcap_{m=1}^{\infty} A^{m}\left(B_{n}\right)$.

Recall the following facts of the sphere $S_{2 n-1}$. (See [6].)

Remarks. 1. There exists exactly one rotation-invariant probability measure $\sigma$ on $S_{2 n-1}$.

2. By use of polar coordinates we identify $S_{2 n-1}$ with the set of angles $\theta:=\left(\theta_{1}, \ldots, \theta_{2 n-1}\right)$, where $\theta$ varies in the closed parallelotope $P$ in $\mathbf{R}^{2 n-1}$ defined by the inequalities

$$
-\frac{1}{2} \pi \leq \theta_{j} \leq \frac{1}{2} \pi \quad(1 \leq j \leq 2 n-2),-\pi \leq \theta_{2 n-1} \leq \pi .
$$

(See [5, p. 174].) Thus we may regard $\sigma$ as defined on $P$. Doing this we obtain the following formula for the Radon-Nikodym derivative

$$
d \sigma=C \cos ^{2 n-2} \theta_{1} \cdot \cos ^{2 n-3} \theta_{2} \cdots \cos \theta_{2 n-2} d m,
$$


where $m$ denotes the Lebesgue measure on $P$, and $C$ is a constant depending on $n$. This formula is easily established, since integration in polar coordinates holds for both measures, see [6, p. 13; 5, p. 177]. Thus we have $\sigma(E)=0$ iff $E$, considered as a set of angles, has Lebesgue measure zero.

The estimates of the stable rank will be obtained by using the following theorem. For functions $g \in A\left(B_{n}\right)$ the zero set is denoted by $Z_{g}$, that is $Z_{g}=\left\{z \in \bar{B}_{n}: g(z)=0\right\}$. The boundary zero set is written as $Z_{\partial}$, i.e., $Z_{\partial}=\left\{z \in \partial B_{n}: g(z)=0\right\}$.

Theorem 2.1. Suppose that each component $f_{j}$ of the vector $f:=\left(f_{1}, \ldots, f_{n}\right)$ is a member of $A^{1}\left(B_{n}\right)$ and that $g \in A\left(B_{n}\right)$ is not identical zero. Then the unbounded open set $\mathbf{C}^{n} \backslash f\left(Z_{g}\right)$ is connected.

Proof. We construct a path $\gamma$ joining the given point $a \in \mathbf{C}^{n} \backslash f\left(Z_{g}\right)$ and infinity. First we show the existence of a cone $K$ with vertex $a$ such that $f\left(Z_{\partial}\right)$ and $K$ are disjoint.

It is well known that $\sigma\left(Z_{\partial}\right)$ is zero, see [6, Theorem 5.5.9]. By Remark 1 the set $Z_{\partial}$, viewed as a set of angles, also has Lebesgue measure zero. Of course we may assume that $a$ is zero, otherwise we deal with the vector $(f-a)$ in the following. Suppose now that $h: P \rightarrow P$ is a $C^{1}$ map, where $P$ denotes the parallelotope mentioned in Remark 2. Then the set $h(E)$ has Lebesgue measure zero for every set $E \subset P$ of Lebesgue measure zero.

The projection $p: Z_{\partial} \rightarrow S_{2 n-1}$ is defined by

$$
p(z):=f(z) /|f(z)| \text {. }
$$

Note that by assumption $0 \notin f\left(Z_{g}\right)$. Let $N \subset P$ be the set of Lebesgue measure zero, where the polar coordinate transform is not injective. Then the set $p\left(Z_{\partial}\right) \backslash N$ is a $C^{1}$ image of a set $E \subset Z_{\partial}$ of Lebesgue measure zero. Thus $p\left(Z_{\partial}\right)$ has Lebesgue measure zero, too. By Remark 2 we have

$$
\sigma\left[p\left(Z_{\partial}\right)\right]=0 .
$$

Since $\sigma\left(S_{2 n-1}\right)=1$, there are many points $\zeta \in S_{2 n-1}$ such that

$$
\zeta \notin p\left(Z_{\partial}\right) \text {. }
$$

Because the set $p\left(Z_{\partial}\right)$ is closed, it is easy to construct a cone $K$ such that $0 \in K$ and $\zeta \in K$. This cone $K$ has the desired property that $K$ and $f\left(Z_{\partial}\right)$ are disjoint.

Now we show that there exists a path $\gamma$ in $K$ joining zero and infinity such that

$$
\gamma \cap f\left(Z_{g}\right)=\varnothing .
$$

Since the cone $K$ misses the points of $f\left(Z_{\partial}\right)$, this is done by showing that $K$ misses the set $f\left(Z_{g} \cap B_{n}\right)$. By [6, Theorem 14.4.9] the set $Z_{g} \cap B_{n}$ is the countable union of compact sets $K_{j}$ with $h_{2 n-2}\left(K_{j}\right)<\infty$. Here, $h_{2 n-2}$ denotes the $(2 n-2)$-dimensional Hausdorff measure (see [6]). Since $f$ is Lipschitz 
continuous in $\bar{B}_{n}$, the same conclusion holds for the set $f\left(Z_{g} \cap B_{n}\right)$. By [6, Theorem 14.4.5] the set $K \backslash f\left(Z_{g} \cap B_{n}\right)$ is connected.

\section{3.}

Let $A$ be a subalgebra of $A\left(B_{n}\right)$. We say that the weak Nullstellensatz holds in $A$ iff the following condition holds: $\left(f_{1}, \ldots, f_{n+1}\right)$ is unimodular iff the functions $f_{1}, \ldots, f_{n+1}$ have no common zero in $\bar{B}_{n}$.

A is said to be inversionally closed if every function $f \in A$ without zero in $\bar{B}_{n}$ is invertible in $A$.

We say that a subalgebra $A$ of $A\left(B_{n}\right)$ is a holomorphically generated $Q$ algebra, for short $H G Q$-algebra, iff the following two conditions hold:

(i) $A$ is a $Q$-algebra.

(ii) There exists a set $L$ of functions holomorphic in $\bar{B}_{n}$ such that $1 \in L$, and in each neighborhood of a suitable element of $A$ we find a member of $L$, that is $\bar{L}=A$.

For example, the ball algebra $A\left(B_{n}\right)$ itself is a $H G Q$-algebra.

The preceding theorem enables us to prove the following results.

Theorem 3.1. Let $A$ be a subalgebra of $A^{1}\left(B_{n}\right)$ in which the weak Nullstellensatz holds. Then we have $\operatorname{sr}(A) \leq n$.

Proof. We must show that every unimodular element $(f, g),\left(f=\left(f_{1}, \ldots, f_{n}\right)\right)$ is reducible. Of course, we may assume that $g$ is not the zero function. Since the weak Nullstellensatz holds in $A$, unimodularity is equivalent to the fact that $f_{1}, \ldots, f_{n}$ and $g$ have no common zero in $\bar{B}_{n}$. Moreover, the algebra $A$ is inversionally closed, so it is a $Q$-algebra, when endowed with the topology of uniform convergence. By Theorem 2.1 the open set $\mathbf{C}^{n} \backslash f\left(Z_{g}\right)$ is connected. Pick $b \notin f\left(\bar{B}_{n}\right)$ and observe that $(f-b, g)$ is reducible. By Proposition 1.1 the element $(f, g)$ is also reducible, since we can join zero and $b$ by a path $\gamma$ lying entirely in $\mathbf{C}^{n} \backslash f\left(Z_{g}\right)$.

Remarks. It should be noted that no assumptions on topology for the algebra $A$ are made.

The fact that $\mathbf{C}^{n} \backslash f\left(Z_{g}\right)$ is connected was used before-implicitly or explicitly - to calculate the stable rank of algebras; see [2 or 3].

Theorem 3.2. Suppose $A \subset A\left(B_{n}\right)$ is a HGQ-algebra in which the weak Nullstellensatz holds. Then the following estimate holds:

$$
\operatorname{sr}(A) \leq n .
$$

Proof. We have to show that every unimodular $(n+1)$-vector $\left(f_{1}, \ldots, f_{n}, g\right)$ is reducible. If $g \equiv 0$, there is nothing to prove, so suppose $g \neq 0$. Since $A$ is a $H G Q$-algebra, every component $f_{j}$ of the vector $\left(f_{1}, \ldots f_{n}\right)$ can be approximated by functions holomorphic in $\bar{B}_{n}$. Because of Proposition 1.2 it is therefore enough to consider the following case: The vector $\left(f_{1}, \ldots, f_{n}, g\right)$ is 
unimodular and each component $f_{j}, j=1, \ldots, n$, is holomorphic in $\bar{B}_{n}$. By Proposition 1.1 it is sufficient to construct a path $\gamma=\left(\gamma_{1}, \ldots, \gamma_{n}\right)$ joining zero and infinity such that every vector $\left(f_{1}-\gamma_{1}(t), \ldots, f_{n}-\gamma_{n}(t), g\right)$ is unimodular. Because of the weak Nullstellensatz this is equivalent to the conclusion

$$
\gamma \subset \mathbf{C}^{n} \backslash f\left(Z_{g}\right),
$$

where we have written $f:=\left(f_{1}, \ldots, f_{n}\right)$. Now, such a path $\gamma$ exists by Theorem 2.1

In general, it is hard to decide whether the weak Nullstellensatz holds or not, so we give the following application of Theorem 3.2. In the sequel $[x]$ denotes the greatest integer less or equal to $x$.

Theorem 3.3. Let $A$ be a subalgebra of the ball algebra $A\left(B_{n}\right)$ which contains all polynomials (in $z_{1}, \ldots, z_{n}$ ) and is inversionally closed. Then the following estimates hold:

$$
[n / 2]+1 \leq \operatorname{sr}(A) \leq n .
$$

Proof. (1) We show that $\operatorname{sr}(A) \leq n$, i.e., every unimodular $(n+1)$-vector $\left(f_{1}, \ldots, f_{n}, g\right)$ is reducible. Because Theorem 3.2 is applicable to the ball algebra $A\left(B_{n}\right)$ itself the vector $\left(f_{1}, \ldots, f_{n}, g\right)$ is reducible in $A\left(B_{n}\right)$, i.e., there exists functions $\alpha_{1}, \ldots, \alpha_{n}, h_{1}, \ldots, h_{n} \in A\left(B_{n}\right)$ such that

$$
\sum_{j=1}^{n} \alpha_{j}\left(f_{j}+h_{j} g\right)=1 \text {. }
$$

Choose polynomials $p_{j}$ such that

$$
\left|h_{j}(z)-p_{j}(z)\right|\left|\alpha_{j}(z) g(z)\right| \leq 1 / 4 n \quad\left(z \in \bar{B}_{n}\right) .
$$

Thus we have

$$
\left|\sum_{j=1}^{n} \alpha_{j}\left(f_{j}+p_{j} g\right)\right| \geq \frac{3}{4} \quad\left(z \in \bar{B}_{n}\right) .
$$

Again we approximate each function $\alpha_{j}$ by polynomials $q_{j}$ :

$$
\left|\alpha_{j}(z)-q_{j}(z)\right|\left|f_{j}(z)+p_{j}(z) g(z)\right| \leq \frac{1}{4} \quad\left(z \in \bar{B}_{n}\right) .
$$

This yields the inequality

$$
\left|\sum_{j=1}^{n} q_{j}\left(f_{j}+p_{j} g(z)\right)\right| \geq \frac{1}{2} \quad\left(z \in \bar{B}_{n}\right) .
$$

Since the algebra $A$ contains all polynomials and is inversionally closed, this inequality shows that $\left(f_{1}, \ldots, f_{n}, g\right)$ is reducible.

(2) We prove that $\operatorname{sr}(A) \geq[n / 2]+1$. Let $k:=[n / 2]$. We consider the $k+1$ polynomials $z_{1}, z_{2}, \ldots, z_{k}$ and $g\left(z_{1}, \ldots, z_{n}\right):=z_{1} z_{k+1}+\cdots+z_{k} z_{2 k}-1$. Obviously, the vector $\left(z_{1}, \ldots, z_{k}, g\right)$ is unimodular. We show that it is not reducible, which settles the assertion. 
To this end we prove that every $k$-vector

$$
\left(z_{1}+h_{1} g, \ldots, z_{k}+h_{k} g\right)
$$

has a common zero in $\bar{B}_{n}$ for suitable $h_{1}, \ldots, h_{k} \in A$. Let $h:=\left(h_{1}, \ldots, h_{k}\right)$, $z:=\left(z_{1}, \ldots, z_{k}\right)$ and define $H: \mathbf{C}^{k} \rightarrow \mathbf{C}^{k}$ by

$$
H(z):= \begin{cases}-h(z, \bar{z}, 0)\left(\left|z_{1}\right|^{2}+\cdots+\left|z_{k}\right|^{2}-1\right), & z \in \bar{B}_{k}, \\ 0 & \text { else. }\end{cases}
$$

Now the function $H$ is continuous and bounded. So there exists a compact disc $K$ such that $H(K) \subset K$. By virtue of the fixed point principle of Brouwer, there exists a fixed point $\zeta$ in $K$, i.e., $H(\zeta)=\zeta$. This equation implies $\zeta \in \bar{B}_{k}$. Written in components the equation $\zeta-H(\zeta)=0$ gives the desired common zero of the vector $\left(z_{1}+h_{1} g, \ldots, z_{k}+h_{k} g\right)$.

Remark. Note that in case $n=2$ we have $\operatorname{sr}(A)=2$, and for $n=1$ we have $\operatorname{sr}(A)=1$.

\section{REFERENCES}

1. H. Bass, K-theory and stable algebra, Publ. Math. I.H.E.S. 22 (1964), 5-60.

2. G. Corach and F. D. Suárez, Extension problems and stable rank in commutative Banach algebras, Topology Appl. 21 (1985), 1-8.

3. _ Stable rank in holomorphic function algebras, Illinois J. Math. 29 (1985), 627-639.

4. - Dense morphisms in commutative Banach algebras, Trans. Amer. Math. Soc. 304 (1987), 537-547.

5. J. Dieudonné, Treatise on analysis, vol. III. Academic Press, New York and London, 1972.

6. W. Rudin, Function theory in the unit ball of $\mathbf{C}^{n}$, Springer-Verlag, New York, 1980.

7. R. Rupp, Über den Bass-Stable-Rank komplexer Funktionen-Algebren, Dissertation, Karlsruhe, 1988.

8. __ Stable rank of holomorphic function algebras, Studia Math. (to appear).

Universität Karlsruhe, Mathematisches Institut I, Englerstr. 2, D-7500 KarlsRuhe 1 